\title{
Polarimetric survey of main-belt asteroids $\mathbf{s}^{\star \star \star}$ IV. New results from the first epoch of the CASLEO survey
}

\author{
R. Gil-Hutton ${ }^{1,2}$, A. Cellino ${ }^{3}$, and Ph. Bendjoya ${ }^{4}$ \\ ${ }^{1}$ Complejo Astronómico El Leoncito (CONICET), Av. España 1512 sur, J5402DSP San Juan, Argentina \\ e-mail: rgilhutton@casleo.gov.ar \\ 2 Universidad Nacional de San Juan, J. I. de la Roza 590 oeste, 5400 Rivadavia, San Juan, Argentina \\ 3 INAF - Osservatorio Astrofisico di Torino, 10025 Pino Torinese, Italy \\ ${ }^{4}$ Université de Nice Sophia Antipolis, Observatoire de la Côte d’Azur, CNRS UMR 7293 Lagrange, Nice, France
}

Received 20 May 2014 / Accepted 7 August 2014

\section{ABSTRACT}

\begin{abstract}
Aims. We present results of a polarimetric survey of main-belt asteroids at Complejo Astronómico El Leoncito (CASLEO), San Juan, Argentina. The aims of this survey are to increase the database of asteroid polarimetry, to estimate diversity in polarimetric properties of asteroids that belong to different taxonomic classes, and to search for objects that exhibit anomalous polarimetric properties.

Methods. The data were obtained using the Torino and CASPROF polarimeters at the $2.15 \mathrm{~m}$ telescope. The Torino polarimeter is an instrument that allows simultaneous measurement of polarization in five different bands, and the CASPROF polarimeter is a two-hole aperture polarimeter with rapid modulation.

Results. The survey began in 1995, and until 2012 data on a large sample of asteroids were obtained. We here present and analyze the unpublished results for 129 asteroids of different taxonomic types, 56 which were polarimetrically observed for the first time. We find that the asteroids (402) Chloe and (729) Watsonia are Barbarians, and asteroid (269) Justitia shows a phase - polarization curve that seems to have a small inversion angle. Data obtained in $U B V R I$ colors allow us to sketch an analysis of the wavelength dependence of the degree of linear polarization for 31 asteroids, in spite of some large error bars in some cases.
\end{abstract}

Key words. minor planets, asteroids: general - techniques: polarimetric

\section{Introduction}

Polarimetry is a very useful observational technique to obtain information on the surface properties of minor bodies. The sunlight scattered by an asteroid surface is in a state of partial linear polarization, which is affected by the composition and roughness of the target. The degree of linear polarization measured by an observer also depends on the illumination conditions. The corresponding variation of polarization with phase angle ${ }^{1}$ (the so-called phase - polarization curve) is described by some parameters that provide information about optical properties of the surface that are related to composition, geometric albedo, rugosity, porosity, etc. (Dollfus et al. 1989; Muinonen et al. 2002; Kaasalainen et al. 2003). The phase - polarization curves of the asteroids display a common general behavior, apart from some differences among different taxonomic classes (Belskaya et al. 2005). As a general rule, there is a so-called branch of negative polarization, which is an interval of phase angles, generally between zero and about 20 degrees, in which the plane of linear polarization is found to be oriented parallel to the scattering plane

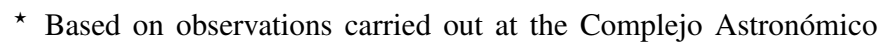
El Leoncito, operated under agreement between the Consejo Nacional de Investigaciones Científicas y Técnicas de la República Argentina and the National Universities of La Plata, Córdoba, and San Juan.

$\star \star$ Table 1 is only available at the CDS via anonymous ftp to cdsarc.u-strasbg.fr (130.79.128.5) or via

http://cdsarc.u-strasbg.fr/viz-bin/qcat?J/A+A/569/A122

1 The angle between the directions from the object to the Sun and the observer. (the plane that contains the Sun, the observer, and the asteroid). The existence of a branch of negative polarization, which contradicts simple expectations based on Fresnel laws, is generally explained in terms of the occurrence of coherent backscattering phenomena (Muinonen et al. 2002, 2007; Tyynelä et al. 2007). The value of polarization corresponding to the deepest point of the negative branch is called $P_{\min }$. Calling $\alpha$ the phase angle, $P_{\text {min }}$ is generally found at $8^{\circ} \lesssim \alpha \lesssim 10^{\circ}$. Beyond an inversion angle $\alpha_{0} \approx 20^{\circ}$, the polarization plane becomes perpendicular to the scattering plane, and we speak about positive polarization.

To obtain good-quality phase - polarization curves of asteroids is challenging. The objects must be observed at several phase angles, and this coverage requires availability of observing time and can be made even more difficult by object faintness, limited visibility, weather problems, etc. As a consequence, the database of asteroid polarimetric measurements has been quite small until about 1990, when very few objects had well determined polarization curves. Since 1995 we have made an extensive effort of polarimetric observations at Complejo Astronómico El Leoncito (CASLEO), Argentina, with the main objective of increasing the available polarimetric database of main-belt asteroids. At the same time, we also developed several observing programs focused on more specific objectives, including studies of the size dependence of the albedo distribution exhibited by the IRAS data (Cellino et al. 1999, 2005a), analyses of the polarimetric properties of different taxonomic classes and asteroid groups (Gil-Hutton 2007; Gil-Hutton et al. 2007; Gil-Hutton \& Cañada-Assandri 2011, 2012; Cañada-Assandri et al. 2012) and of some individual targets, like the near-Earth 
asteroid (25453) Itokawa, the target of the Japanese Hayabusa space mission (Cellino et al. 2005b). An unexpected result of this overall observational effort was the discovery of the unusual polarimetric behavior of some objects, the so-called Barbarians (Cellino et al. 2006; Gil-Hutton et al. 2008).

In this paper we report polarimetric observations obtained during the past 18 years that were not yet published. In Sect. 2 we describe the observations, in Sect. 3 we present and discuss our results, and in Sect. 4 we sketch some conclusions and future work.

\section{Observations}

Our observations were carried out during different observing runs between February 1995 and July 2012 at the 2.15 m telescope of the CASLEO, using either the Torino or the CASPROF polarimeters. A full description of the Torino photopolarimeter can be found in Piirola (1988) and Scaltriti et al. (1989), while a description of CASPROF is given in Gil-Hutton et al. (2008). From the analysis of several standard stars, we found the instrumental polarization to be fairly constant and stable, always below $0.1 \%$ for both instruments and for each filter.

Whenever possible, we observed the targets during runs some weeks apart to obtain measurements during the same apparition at different phase angles. Observing nights were generally assigned around the new Moon to minimize the contamination of sky polarization by moonlight, mainly when we used the CASPROF polarimeter. In all cases, we used the smallest diaphragm allowed by the observing conditions to minimize the contribution of sky background (moreover, the design of the Torino polarimeter was such as to minimize a priori the effect of sky background contamination, Piirola 1988) and took sky measurements frequently to test for any variation. Each night, we observed at least two zero-polarization standard stars and one highpolarization star to determine instrumental polarization. The standard star data were obtained from Turnshek et al. (1990), Gil-Hutton \& Benavidez (2003), and Fossati et al. (2007).

We observed the targets consecutively several times each night with individual exposure times long enough to reach final signal-to-noise ratios $(S / N) \geq 100$ for different filters (at least $90 \mathrm{~s}$ and $180 \mathrm{~s}$ for the Torino and CASPROF polarimeters, respectively). Several measurements of each object were co-added to improve the $\mathrm{S} / \mathrm{N}$, and the measurement errors were evaluated assuming a Poisson distribution. After correcting for instrumental polarization, we obtained the Stokes parameters for each filter with reduction programs specifically designed for each instrument, with some modifications to optimize the reduction to the specific needs of asteroid polarimetry. This included computations of the position angle of the scattering plane and of the polarization parameter $P_{r}=\left(I_{\perp}-I_{\|}\right) /\left(I_{\perp}+I_{\|}\right)$, where $I_{\perp}$ and $I_{\|}$are the intensities of the scattered light polarized along the planes perpendicular and parallel to the scattering plane, respectively. The overall data reduction pipeline is essentially identical to that previously used in Cellino et al. (1999, 2005a, 2006), Gil-Hutton (2007), and Gil-Hutton \& Cañada-Assandri (2011). As a test of the data reduction process, a histogram of the ratio between the $U$ component of linear polarization and its error was made, and the distribution is to be centered at zero and all points differ from this value by less than $3-4 \sigma$, which supports the reliability of the polarimetric measurements.

\section{Results}

During this survey we obtained 932 observations of 129 mainbelt asteroids in different filters (145 observations in $U, 145$ in $B$,
342 in $V, 158$ in $R$, and 142 in $I$ ), which almost doubles the currently available database at the Planetary Data System (PDS) ${ }^{2}$. Table 1 lists the asteroid number, the observing date, the phase angle $(\alpha)$, the polarization and position angle with respect to the scattering plane for each filter, and its Bus taxonomic classification (Bus 1999) taken from Bus \& Binzel (2002), Lazzaro et al. (2004) or the PDS. The taxonomic classification, which is based on measurements of spectral reflectance data mostly at visible wavelengths, is a discipline that has evolved with time. We used here the Bus classification (Bus \& Binzel 2002), since it is one of the most recent and widely adopted ones. We should bear in mind some caveats, however. In particular, the modern taxonomic classifications, including the one by Bus \& Binzel (2002), are based on campaigns of spectroscopic observations making use of CCD detectors that do not cover adequately the blue part of the reflectance spectrum. The older spectrophotometric surveys were based on photomultipliers that, although undoubtedly performed less well, also covered the $U$ and $B$ standard colors. As a consequence, some classes that were kept separate in earlier taxonomic classifications, like the old F- and B-types found in the classical analysis of Tholen (1989), are now merged into a unique B-class in the more recent Bus \& Binzel (2002) classification. We show below that polarimetry strongly suggests that this merging of the old F- and B-classes is incorrect, because the polarimetric properties of the objects appear to be clearly different. We also note that the so-called X-complex in the Bus classification is subdivided into different classes (X-, Xe-, Xc-, and $\mathrm{Xk}$-) that are known to include objects that are quite different in terms of albedo and composition (the old E-, M- and Pclasses found by Tholen 1989). Therefore, we can a priori expect that the polarimetric behavior of objects belonging to the modern X-complex will be found to be quite heterogeneous, because the degree of linear polarization does depend on the albedo.

Since many observations listed in Table 1 were obtained simultaneously in $U B V R I$ bands using the Torino polarimeter, these data allow us to attempt an analysis of the wavelength dependence of linear polarization for 31 asteroids observed at 44 different phase angles. In particular, taking into account that the degree of linear polarization is simply the module of $P_{r}$, we were able to compute a linear spectro-polarimetric slope parameter $\left|\Delta P_{r}\right| / \Delta \lambda$ in a wavelength range of $0.37-0.83 \mu \mathrm{m}$.

We note that our results are not immediately comparable with those of Belskaya et al. (2009) since these authors chose to plot as a function of wavelength the value of $P_{r}$, which is the degree of linear polarization with a sign to distinguish between negative and positive polarization. We here prefer to obtain the spectro-polarimetric slope parameter using the degree of linear polarization (equal to the module of $P_{r}$ ) because we think that this parameter is more useful, and also to avoid some possible complications in the interpretation of the spectro-polarimetric slope, that are related to the sign of $P_{r}$.

To try to avoid exceedingly large errors, we only considered data with nominal errors of polarization below $15 \%$, but in a few cases we accepted larger errors for observations in the $U$ filter. We stress that as a general rule, our polarimetric measurements had relatively small error bars only in $V$ and $R$ colors. In $U, B$ and $I$ bands the error bars tend to be larger, even much larger especially in $U$, as a general rule, because in these bands we obtain much fewer photons. For this reason, we clearly state that the data listed in Tables 2, 3, and 4 should only be interpreted in a statistical sense, not as detailed results obtained for each individual object, since they may be very inaccurate in many

Available at the URL http://sbn.psi.edu/pds/asteroid 
R. Gil-Hutton et al.: Polarimetric survey of main-belt asteroids. IV.

Table 2. Spectro-polarimetric slope parameters for S-complex, L-, and K-type asteroids.

\begin{tabular}{rrrrl}
\hline \hline & $\begin{array}{c}\alpha \\
\mathrm{deg}\end{array}$ & $\begin{array}{c}\text { Slope } \\
\% / \mu \mathrm{m}\end{array}$ & $\sigma$ & Tax \\
\hline 6 & 15.16 & -0.060 & 0.185 & $\mathrm{~S}$ \\
8 & 24.51 & -0.794 & 0.315 & $\mathrm{~S}$ \\
8 & 30.91 & -0.849 & 0.208 & $\mathrm{~S}$ \\
8 & 31.52 & -0.827 & 0.445 & $\mathrm{~S}$ \\
18 & 28.00 & -0.497 & 0.206 & $\mathrm{~S}$ \\
27 & 9.94 & -0.291 & 0.265 & $\mathrm{~S}$ \\
27 & 11.63 & -0.286 & 0.192 & $\mathrm{~S}$ \\
118 & 9.32 & 0.013 & 0.277 & $\mathrm{~S}$ \\
183 & 8.49 & 0.321 & 0.290 & $\mathrm{~S}$ \\
183 & 9.19 & 0.262 & 0.242 & $\mathrm{~S}$ \\
192 & 26.57 & -2.267 & 0.234 & $\mathrm{~S} 1$ \\
230 & 7.40 & -0.266 & 0.240 & $\mathrm{Sl}$ \\
\hline 12 & 14.70 & 0.796 & 0.265 & $\mathrm{~L}$ \\
12 & 14.99 & 1.090 & 0.324 & $\mathrm{~L}$ \\
219 & 28.83 & -0.752 & 0.589 & $\mathrm{~L}$ \\
269 & 28.42 & -2.651 & 1.611 & $\mathrm{Ld}$ \\
269 & 28.49 & -3.637 & 1.060 & $\mathrm{Ld}$ \\
\hline 221 & 10.14 & 0.241 & 0.394 & $\mathrm{~K}$ \\
221 & 9.53 & 0.421 & 0.368 & $\mathrm{~K}$ \\
\hline
\end{tabular}

Notes. The columns list the asteroid number, phase angle, slope parameter and its error, and Bus taxonomic type.

Table 3. Same as Table 2, but for C-complex and B-type asteroids.

\begin{tabular}{rrrll}
\hline \hline & $\begin{array}{c}\alpha \\
\mathrm{deg}\end{array}$ & $\begin{array}{c}\text { Slope } \\
\% / \mu \mathrm{m}\end{array}$ & $\sigma$ & Tax \\
\hline 1 & 7.30 & 0.194 & 0.121 & $\mathrm{C}$ \\
10 & 15.50 & -0.625 & 0.104 & $\mathrm{C}$ \\
19 & 19.00 & -0.855 & 0.569 & $\mathrm{Ch}$ \\
41 & 8.41 & -0.720 & 0.593 & $\mathrm{Ch}$ \\
91 & 3.00 & 0.066 & 0.125 & $\mathrm{Ch}$ \\
154 & 9.90 & 0.118 & 0.735 & $\mathrm{C}$ \\
253 & 30.19 & 0.828 & 1.037 & $\mathrm{Cb}$ \\
350 & 16.40 & -0.334 & 0.503 & $\mathrm{Ch}$ \\
419 & 11.86 & -2.005 & 0.592 & $\mathrm{Cb}$ \\
\hline 2 & 14.60 & 0.138 & 0.050 & $\mathrm{~B}$ \\
335 & 10.03 & -0.893 & 0.578 & $\mathrm{~B} *$ \\
335 & 10.90 & -0.791 & 0.374 & $\mathrm{~B} *$ \\
372 & 10.16 & -0.479 & 0.398 & $\mathrm{~B}$ \\
372 & 9.85 & -0.021 & 0.364 & $\mathrm{~B}$ \\
431 & 8.12 & -0.632 & 0.412 & $\mathrm{~B}$ \\
431 & 9.37 & -0.471 & 0.397 & $\mathrm{~B}$ \\
704 & 8.20 & -0.495 & 0.182 & $\mathrm{~B} *$ \\
\hline
\end{tabular}

Notes. The B-types marked with an asterisk are objects classified as F-types by Tholen.

cases, as shown by the listed values of $\sigma$ of the obtained spectropolarimetric slopes. It is more useful to compare the differences among the average trends that can be found among objects that belong to different taxonomic classes.

The asteroid number, phase angle, slope parameter and its error, and taxonomic type for asteroids with taxonomic classification in the S-, C-, and X-complexes of Bus (1999) and Bus \& Binzel (2002) are listed in Tables 2-4, respectively. In addition to the large relative errors of the derived slopes, which are a consequence of the error bars of individual measurements, as mentioned above, some discrepant behavior among objects within the same taxonomic class can in principle be also a consequence of grouping together objects that may be intrinsically quite different. We show below that this seems to be clearly true for the merging of C-, B- and F-class objects. This is also clearly true
Table 4. Same as Table 2, but for X-complex asteroids.

\begin{tabular}{rrrcl}
\hline \hline & $\begin{array}{c}\alpha \\
\mathrm{deg}\end{array}$ & $\begin{array}{c}\text { Slope } \\
\% / \mu \mathrm{m}\end{array}$ & $\sigma$ & $\mathrm{Tax}$ \\
\hline 21 & 4.50 & 0.497 & 0.224 & $\mathrm{Xk}$ \\
21 & 27.87 & -0.177 & 0.456 & $\mathrm{Xk}$ \\
22 & 12.49 & 0.262 & 0.470 & $\mathrm{X}$ \\
22 & 13.25 & 0.326 & 0.200 & $\mathrm{X}$ \\
71 & 6.66 & -0.077 & 0.108 & $\mathrm{Xe}$ \\
161 & 27.59 & -3.003 & 0.751 & $\mathrm{Xc}$ \\
161 & 27.80 & -3.197 & 0.780 & $\mathrm{Xc}$ \\
268 & 6.90 & 0.464 & 0.294 & $\mathrm{X}$ \\
\hline
\end{tabular}

for the X-complex, because we know a priori that the $\mathrm{X}$-complex includes objects with very different albedos.

Taking all these caveats in mind, and calling that the inversion angle for most asteroids is normally around 20 degrees, we find that objects characterized by moderate values of albedo, mostly belonging to the S-complex (Table 2), tend to have a negative spectro-polarimetric slope when observed in the positive branch of polarization. In other words, at large phase angles these objects clearly tend to be more polarized in the blue part of the spectrum than in the red part. In the negative polarization branch the situation is more complicated, but it seems that most objects tend to have a flat or positive spectro-polarimetric slope. This means that at small phase angles these objects tend to be more polarized in the red region of the spectrum than in the blue. Exceptions appear to be present, but the general trend is the one just mentioned. The behavior exhibited by low-albedo asteroids (Table 3 ) clearly shows that apart from a few isolated cases, the behavior seems to be exactly the opposite: the spectropolarimetric slope tends to be negative in the negative polarization branch, and positive at large phase angles. This general difference in the behavior of S- and C-class asteroids has previously been noticed by Belskaya et al. (2009), and we fully confirm it.

Table 4 shows that the few data at our disposal suggest that (21) Lutetia, for a long time classified as M-class, behaviors similarly to moderate albedo asteroids (negative slope at large phase angles, and positive slope in the negative polarization branch). The Xc asteroid (161) Athor, previously classified as $\mathrm{M}$, which is observed at large phase angles, also shows a negative spectral slope. The X class objects (22) Kalliope (previously classified as M) and (268) Adorea (previously classified as FC, namely as a dubious case, probably low-albedo), both observed in the negative polarization branch, exhibit a positive spectropolarimetric slope, again similar to the behavior of moderatealbedo objects. The Xe asteroid (71) Niobe, however, previously classified as $\mathrm{S}$, shows a mildly negative slope in the negative polarization branch.

The situation seems fairly complicated. However, the opposite behavior displayed by objects belonging to the $S$ - and C-complexes appears to be fairly sharp and interesting, which may be related to the role played by the Umov effect and multiple scattering on surfaces of different albedo. These data seem to be an important constraint for physical models of light scattering by planetary surfaces. The situation seems to be even more complicated for the objects belonging to the X-complex, probably because of the intrinsic heterogeneity of this taxonomic complex.

Figure 1 shows 183 observations of 37 S-complex asteroids in $V$ and $R$ bands, where the phase - polarization curve obtained by Gil-Hutton \& Cañada-Assandri (2011; using data that were primarily in $V$ color, plus a smaller number of observations in $R$ ) is plotted for a comparison. In this plot, the scatter of the points 


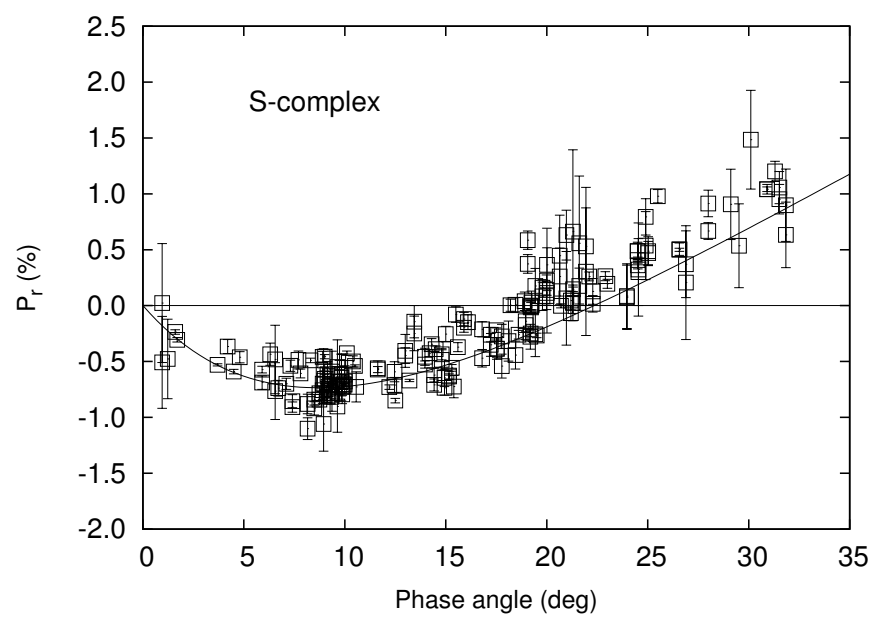

Fig. 1. Polarimetric observations of main-belt asteroids belonging to the S-complex. The average phase-polarization curve for the S-class found by Gil-Hutton \& Cañada-Assandri (2011) is also shown.

may in part be due to a slightly systematic difference between data in $V$ and $R$ colors, as we showed above, and in part to merging some different spectroscopical subsets of the S-complex (S, Sa, Sl, and Sk from Bus \& Binzel 2002), which may have slightly different properties. The small group of observations that in the plot appear to be concentrated around $\alpha \simeq 20^{\circ}$ and have $P \simeq 0.5 \%$, clearly above the average phase - polarization curve, refer to one individual object, the asteroid (502) Sigune. These data are quite noisy, but we decided to keep them in the figure since it is difficult to decide whether we have clear evidence of a peculiar behavior, or only poor-quality data.

Figure 2 shows 36 observations of $8 \mathrm{~L}$ - and Ld-type asteroids in $V$ and $R$ bands, where the phase - polarization curve obtained by Gil-Hutton \& Cañada-Assandri (2011) for L-type is also included for comparison. Observations of the asteroids (172) Baucis, (234) Barbara, and (980) Anacostia, are included in that figure although they were previously published, are included in that figure. These objects, commonly known as Barbarians, from their prototype (234) Barbara, were found by Cellino et al. (2006) and Gil-Hutton et al. (2008) to show an unusual polarimetry behavior characterized by an inversion angle $\alpha_{0} \simeq 30^{\circ}$. Although (234) Barbara itself is classified as Ld-class (which might suggest that this class might be a good candidate to include all Barbarians), there are other known Ld asteroids, such as (269) Justitia, which are not Barbarians, as shown in Fig. 2. It is interesting to see that (269) Justitia instead shows a phase polarization curve characterized by a fairly small inversion angle $\alpha_{0} \simeq 15^{\circ}$, which seems a commona property among asteroids previously classified by Tholen (1989) as F-type (Belskaya et al. 2005). Of course, we cannot exclude that some misclassification might be present in the Bus \& Binzel (2002) taxonomic catalogue, mostly in cases for which it may be difficult to distinguish between objects belonging to taxonomic classes that show fairly similar spectral properties. In this respect, we note that (980) Anacostia and (729) Watsonia, which have both been found to be Barbarians based on our CASLEO observations (see also Masiero \& Cellino 2009), have been classified by Bus \& Binzel (2002) as L-class, not Ld. It is also interesting to note that (729) Watsonia has been found to be the lowest-numbered member of a dynamical family (Novaković et al. 2011), which has been recently found to include several newly discovered Barbarian objects (Cellino et al. 2014). (980) Anacostia is also very close and possibly related to this Watsonia family.

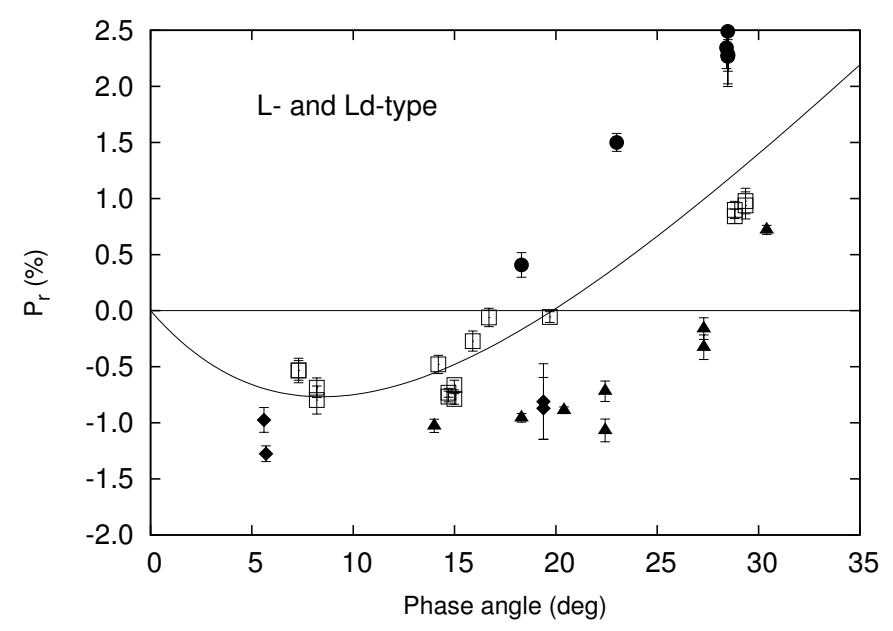

Fig. 2. Polarimetric observations of L- and Ld-class main-belt asteroids. The observations indicated by squares are measurements of L-class objects, those indicated by filled circles are measurements of the Ld-class object (269) Justitia. The observations indicated by filled triangles are from objects classified as Barbarians, and those indicated by diamonds are measurements of the object (729) Watsonia, which is as well a Barbarian. The phase-polarization curve for L-class objects found by Gil-Hutton \& Cañada-Assandri (2011) is also shown.

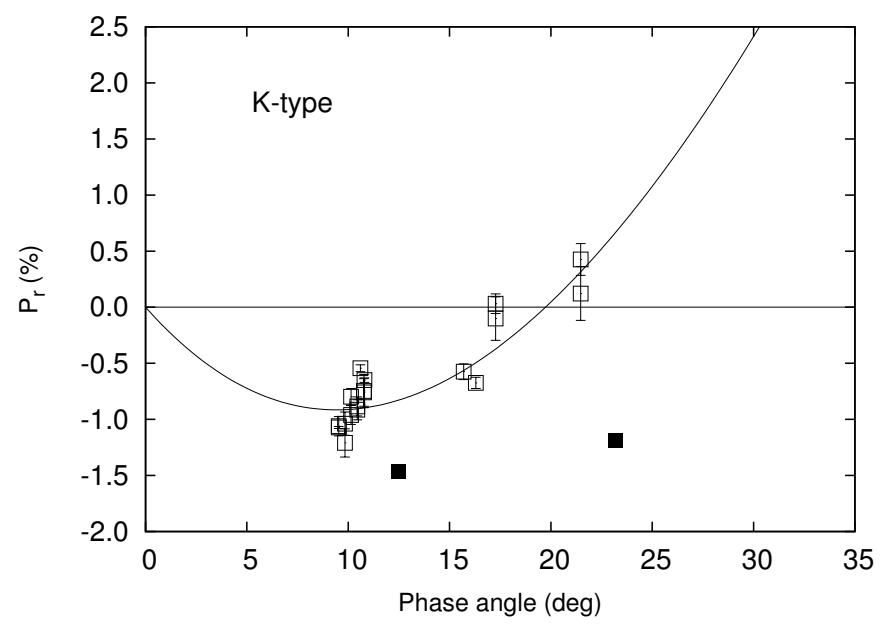

Fig. 3. Polarimetric observations of K-class main-belt asteroids. The observations indicated by filled squares are measurements of the object (402) Chloe. The phase-polarization curve for these taxonomic class found by Cañada-Assandri et al. (2012) is also shown.

Twenty observations of five K-type asteroids in $V$ and $R$-bands are shown in Fig. 3. All the observations follow the phase - polarization curve proposed by Cañada-Assandri et al. (2012) with the exception of those of the asteroid (402) Chloe, which suggests a large inversion angle, indicating that this object must be added to the list of known Barbarians. Chloe belongs to the K-class but we know that there is at least another asteroid classified as K, (679) Pax, which is also a Barbarian (Gil-Hutton et al. 2008).

Eighty-six observations of 33 asteroids belonging to the $\mathrm{C}$-, $\mathrm{Cb}$-, and Ch-types were obtained in this survey (Fig. 4). The average phase - polarization curves found by Gil-Hutton \& Cañada-Assandri (2012) for these taxonomic types are also shown in the plots. The agreement is good only for the Ch-type. These new polarimetric measurements for the C-types seem to be systematically above the curve that was found by considering a different data set. Again, the apparent discrepancies might 
R. Gil-Hutton et al.: Polarimetric survey of main-belt asteroids. IV.
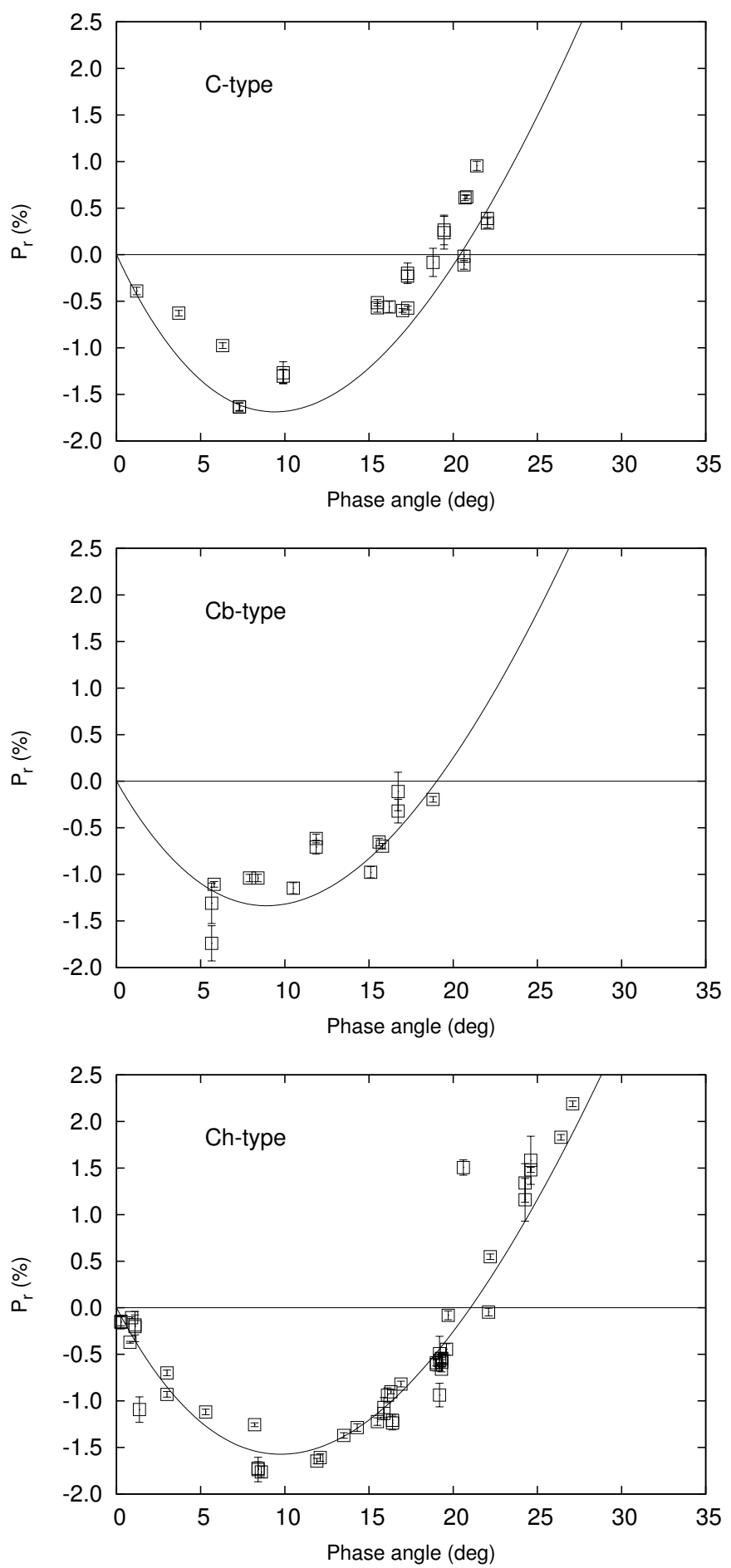

Fig. 4. Polarimetric observations of $\mathrm{C}-, \mathrm{Cb}-$, and Ch-class main-belt asteroids. The phase-polarization curves for these taxonomic types found by Gil-Hutton \& Cañada-Assandri (2012) are also shown.

be indicate some heterogeneity within the different classes included in the $\mathrm{C}$ complex, or alternatively, there might be some misclassification of the objects.

The data well above the curve at $\alpha \simeq 12^{\circ}$ in the Cb-class plot come from a measurement of the asteroid (419) Aurelia, which is known to show an inversion angle of $\approx 15^{\circ}$ (Belskaya et al. 2002), typical of the old F-class of Tholen, and does not follow the general polarimetric behavior of $\mathrm{Cb}$ objects. The very high polarization measurement at $\alpha \simeq 20^{\circ}$ in the $\mathrm{Ch}$ plot refers to

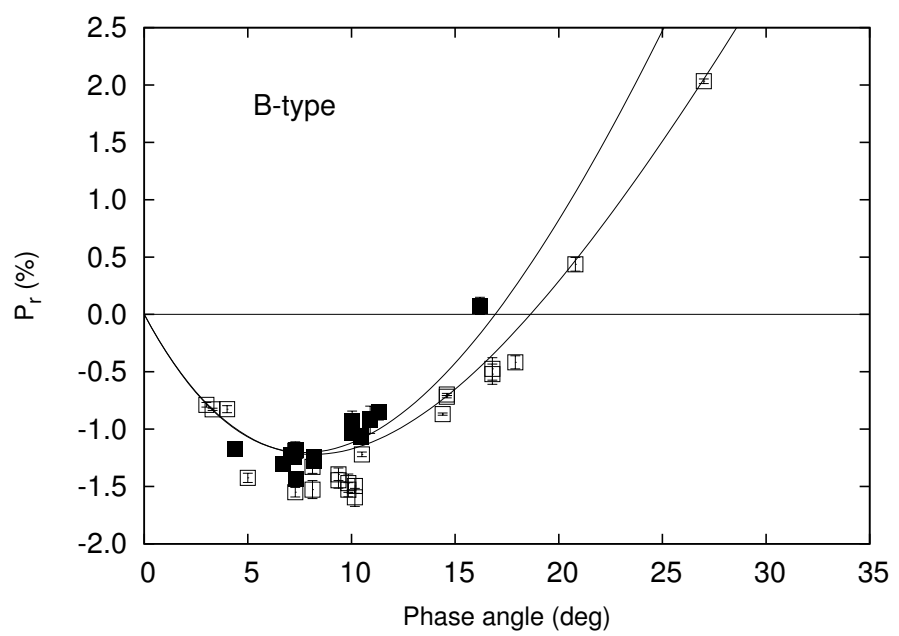

Fig. 5. Polarimetric observations of main-belt asteroids classified as B by Bus \& Binzel (2002). The observations indicated by filled squares are measurements of objects that show a small inversion angle. The phase-polarization curves for asteroids with or without small inversion angle found by Gil-Hutton \& Cañada-Assandri (2012) are also shown.

(238) Hypathia and might be indicate an unusual polarimetric behavior for this asteroid, which deserves confirmation.

Thirty-eight observations of seven B-class asteroids in $V$ and $R$ bands were obtained (Fig. 5). These data show some scattering at phase angles in the range $10^{\circ} \lesssim \alpha \lesssim 18^{\circ}$, and strongly suggest an overall bimodality. The reason is probably that the asteroids classified by Bus \& Binzel (2002) as members of the B-class include objects that belong to two distinct classes. In particular, the old F-class of Tholen, which was previously distinct from the B-class defined by the same author, is now merged into the new B-class of Bus \& Binzel. However, it has been shown that the objects previously classified as $\mathrm{F}$ are characterized by a well-defined polarimetric behavior, whose primary characteristic is a comparatively low value of the inversion angle (Belskaya et al. 2005). This is the case, for instance, of the asteroids (335) Roberta and (704) Interamnia included in (Fig. 5). The previously mentioned asteroid (419) Aurelia, currently classified as Cb-type, was also previously classified as $\mathrm{F}$ by Tholen (1989), and it also has a rather low value of the inversion angle. This is a good example of the effectiveness of polarimetry as a valid tool for taxonomic classification purposes, which complements asteroid spectroscopy well.

Ninety observations of $23 \mathrm{X}-, \mathrm{Xc-}, \mathrm{Xk}-$, and Xe-class asteroids in $V$ and $R$ bands are shown in Fig. 6. As mentioned above and as also noted by Cañada-Assandri et al. (2012), the high dispersion of the data is a result of merging objects with quite heterogeneous surface properties, but all included in the Bus \& Binzel X-complex. Some of these objects were previously assigned to different classes (E-, M-, P-types) by Tholen (1989), and were known to be strongly different in terms of albedo (high, moderate, and low for E, M, and P, respectively). Polarimetry is strongly sensitive to albedo differences. As a reference, the phase - polarization curves for moderate and low albedo found by Cañada-Assandri et al. (2012) are also included in Fig. 6. We here have again proof of the effectiveness of polarimetry in distinguishing bodies with different surface properties (primarily the albedo) among a complex of objects that are merged, from spectroscopic data that cover visible wavelengths alone. We are aware that spectroscopy can also distinguish among different 


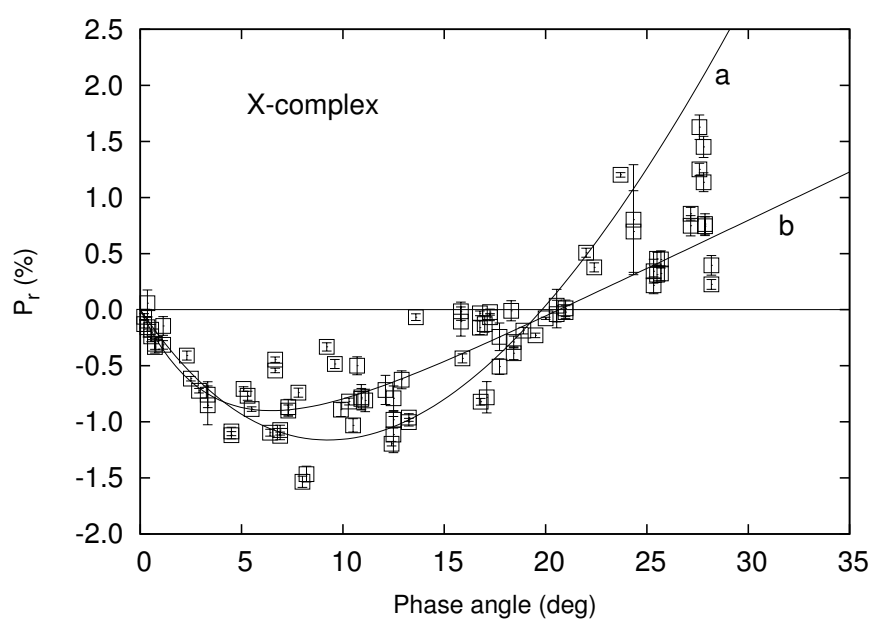

Fig. 6. Polarimetric observations of X-complex main-belt asteroids. Phase-polarization curves for asteroids belonging to the old $\mathrm{P}$ - and M-classes of Tholen (1989) found by Gil-Hutton \& Cañada-Assandri (2011) are indicated with a and b, respectively.

subclasses of the $\mathrm{X}$ complex, but only when near-IR spectroscopic data are available.

Among the 129 asteroids observed in this survey, we report the first polarimetric observations for 56 objects that belong to a wide variety of taxonomic classes: (72) Feronia, (73) Klytia, (91) Aegina, (94) Aurora, (105) Artemis, (113) Amalthea, (118) Peitho, (123) Brunhild, (131) Brunhildala, (134) Sophrosyne, (138) Tolosa, (154) Bertha, (158) Koronis, (169) Zelia, (183) Istria, (186) Celuta, (188) Menippe, (189) Phthia, (197) Arete, (200) Dynamene, (204) Kallisto, (222) Lucia, (226) Weringia, (238) Hypatia, (243) Ida, (246) Asporina, (268) Adorea, (288) Glauke, (308) Polyxo, (317) Roxane, (341) California, (350) Ornamenta, (359) Georgia, (368) Haidea, (376) Geometria, (444) Gyptis, (502) Sigune, (504) Cora, (564) Dudu, (573) Recha, (578) Happelia, (660) Crescentia, (678) Fredegundis, (725) Amanda, (729) Watsonia, (732) Tjilaki, (779) Nina, (823) Sisigambis, (876) Scott, (914) Palisana, (1166) Sakuntala, (1366) Piccolo, (2209) Tianjin, (3353) Jarvis, (3906) Chao, and (5111) Jacliff.

\section{Conclusions and future work}

Using the Torino and the CASPROF polarimeters at Complejo Astronómico El Leoncito, we obtained 932 polarimetric measurements in several bands for 129 main-belt asteroids of different taxonomic types, 56 of them polarimetrically observed for the first time.

The observational data allowed us to preliminarily analyze the wavelength dependence of the polarization for 31 asteroids at different phase angles. We found systematic differences in the observed behavior of the S- and C-complexes, that is, between objects with moderate and low albedo.

The data obtained in this survey agree generally well with the average phase - polarization curves found by Gil-Hutton \& Cañada-Assandri (2011, 2012), and Cañada-Assandri et al. (2012) for different taxonomic classes. CASLEO observations were crucial for the discovery of the Barbarians, including the new identification of (402) Chloe and (729) Watsonia as Barbarians, which was presented for the first time in this paper. Other cases of interesting polarimetric properties still deserve confirmation, for example, L-type asteroid (269) Justitia and the
Ch-type asteroid (238) Hypatia. This might lead us to new interesting discoveries.

The polarimetric observations carried out at CASLEO certainly prove the effectiveness of polarimetry as a tool for asteroid taxonomy. In particular, the capability of distinguishing between objects with different albedos and/or that belong to taxonomic classes that are hardly distinguishable today based on visible spectroscopic data, is certainly a major merit of asteroid polarimetry.

The Torino and CASPROF polarimeters are no longer offered to observing proposals at the $2.1 \mathrm{~m}$ CASLEO telescope. This means that the CASLEO polarimetric survey as it was managed in the past has reached an end. Since the last semester of 2013, CASLEO has offered a new polarimeter, called CASPOL, based on a much better CCD detector, which allows us to observe fainter objects. The second epoch of this survey will be based on this new equipment, and its main objective will be to observe fainter asteroids, study the polarimetric properties of families and groups, and try to detect new cases of anomalous polarimetric behavior.

Acknowledgements. The authors thank the referee Karri Muinonen for his review, which led to an improvement of the paper. R.G.H. gratefully acknowledges financial support by CONICET through PIP 114-200801-00205 and PIP 114-201101-00358.

\section{References}

Belskaya, I. N., Shevchenko, V. G., Efimov, Yu. S., et al. 2002, in Proc. of Asteroids, Comets, Meteors, ed. B. Warmbein (Berlin: ESA Publishing Division), 489

Belskaya, I. N., Shkuratov, Yu. G., Efimov, Yu. S., et al. 2005, Icarus, 178, 213

Belskaya, I. N., Levasseur-Regourd, A-.C., Cellino, A., et al. 2009, Icarus, 199, 97

Bus, S. J. 1999. Ph.D. Thesis, Massachusetts Institute of Technology, Boston Bus, S. J., \& Binzel, R. P. 2002, Icarus, 158, 146

Cañada-Assandri, M., Gil-Hutton, R., \& Benavidez, P. 2012, A\&A, 542, A11

Cellino, A., Gil-Hutton, R., Tedesco, E. F., Di Martino, M., \& Brunini, A. 1999, Icarus, 138, 129

Cellino, A., Gil-Hutton, R., Di Martino, M., et al. 2005a, Icarus, 179, 304

Cellino, A., Yoshida, F., Anderlucci, E., et al. 2005b, Icarus, 179, 297

Cellino, A., Belskaya, I. N., Bendjoya, Ph., et al. 2006, Icarus, 180, 565

Cellino, A., Bagnulo, S., Tanga, P., Novaković, B., \& Delbò, M. 2014, MNRAS, 439, L75

Dollfus, A., Wolff, M., Geake, J. E., Lupishko, D. F., \& Dougherty, L. 1989, in Asteroids II, eds. R. P. Binzel, T. Gehrels, \& M. S. Matthews (Tucson: Univ. of Arizona Press), 594

Fossati, L., Bagnulo, S., Mason, E., \& Landi Degl'Innocenti, E. 2007, ASP Conf. Ser., 364, 503

Gil-Hutton, R. 2007, A\&A, 464, 1127

Gil-Hutton, R., \& Benavidez, P. 2003, MNRAS, 345, 97

Gil-Hutton, R., Lazzaro, D., \& Benavidez, P. 2007, A\&A, 468, 1109

Gil-Hutton, R., Mesa, V., Cellino, A., et al. 2008, A\&A, 482, 309

Gil-Hutton, R., \& Cañada-Assandri, M. 2011, A\&A, 529, A86

Gil-Hutton, R., \& Cañada-Assandri, M. 2012, A\&A, 539, A115

Kaasalainen, S., Piironen, J., Kaasalainen, M., et al. 2003, Icarus, 161, 34

Lazzaro, D., Angeli, C. A., Carvano, J. M., et al. 2004, Icarus, 172, 179

Masiero, J., \& Cellino, A. 2009, Icarus, 199, 333

Muinonen, K., Piironen, J., Shkuratov, Yu. G., Ovcharenko, A., \& Clark, B. E. 2002, in Asteroids III, eds. W. F. Bottke jr., A. Cellino, P. Paolicchi, \& R. P. Binzel (Tucson: Univ. of Arizona Press), 123

Muinonen, K., Zubko, E., Tyynelä, J., Shkuratov, Yu. G., \& Videen, G. 2007, J. Quant. Spectrosc. Radiat. Transfer., 106, 360

Novaković, B., Cellino, A., \& Kneżević, Z. 2011, Icarus, 216, 69

Piirola, V. 1988, in Polarized Radiation of Circumstellar Origin, eds. G. V. Coyne, et al. (Tucson: Univ. of Arizona Press), 735

Scaltriti, F., Piirola, V., Cellino, A., et al. 1989, Mem. Soc. Astron. It., 60, 243

Tholen, D. J. 1989, in Asteroids II, eds. R. P. Binzel, T. Gehrels, \& M. S. Matthews (Tucson: Univ. of Arizona Press), 1139

Turnshek, D. A., Bohlin, R. C., Williamson II, R. L., Lupie, O. L., \& Koornneef, J. 1990, AJ, 99, 1243

Tyynelä, J., Zubko, E., Videen, G., \& Muinonen, K. 2007, J. Quant. Spectrosc. Radiat. Transf., 106, 520 